\title{
Purification and Characterization of Macrophage Migration Inhibitory Factor as a Secretory Protein from Rat Epididymis: Evidences for Alternative Release and Transfer to Spermatozoa
}

\author{
Regina Eickhoff, ${ }^{1}$ Beate Wilhelm, ${ }^{1}$ Heiner Renneberg, ${ }^{1}$ Gunther Wennemuth, ${ }^{1}$ Michael Bacher, ${ }^{2}$ \\ Dietmar Linder, ${ }^{2}$ Richard Bucala, ${ }^{3}$ Jürgen Seitz $^{1}$ and Andreas Meinhardt ${ }^{1}$ \\ ${ }^{1}$ Department of Anatomy and Cell Biology and ${ }^{2}$ Department of Immunology, \\ Philipps University of Marburg, Marburg, Germany \\ ${ }^{2}$ Department of Biochemistry, Justus Liebig-University, Giessen \\ ${ }^{3}$ The Picower Institute for Medical Research, Manhasset, New York, U.S.A. \\ Communicated by R. Bucala. Accepted September 15, 2000.
}

\begin{abstract}
Background: The cytokine macrophage migration inhibitory factor (MIF), originally described as a T cell product, has recently been identified to mediate cellular interactions in several endocrine organs. Western blots analysis of rat epididymal homogenates using an anti-MIF antibody indicated the presence of substantial amounts of an immunoreactive protein with the apparent $M_{\mathrm{r}}$ of $12 \mathrm{kDa}$. Our study aimed to characterize the molecular nature of this immunoreactive factor.

Materials and Methods: The purified $12 \mathrm{kDa}$ protein and a cloned cDNA fragment were characterized by sequence analysis. Furthermore, expression pattern and localization of the $12 \mathrm{kDa}$ protein were investigated using in situ hybridization, immunohistochemistry, immunoelectron microscopy, and western blots experiments on epididymal sections, isolated epididymal vesicles, and outer dense fibers from spermatozoa.

Results: The N-terminal amino acid sequence analysis over 10 amino acids revealed a $100 \%$ homology of the $12 \mathrm{kDa}$ protein to the $\mathrm{N}$-terminus of the cytokine MIF. These data were confirmed by sequence analysis of a reverse transcription polymerase chain reaction (RT-PCR) amplified cDNA fragment from rat epididymis, which also showed complete homology to the MIF cDNA sequence.
\end{abstract}

MIF protein and mRNA were localized in the epithelial cells of the epididymis in a regional distribution manner, with the expression maximal in the caput. Immune cells were not labeled. MIF is the first classical cytokine identified to be expressed by the epididymal epithelial cells. Immunoelectron microscopy detected MIF immunoreactivity in the cytoplasm, with no reaction visible in the Golgi complex and the cisternae of the endoplasmic reticulum. At the apical cell surface, MIF accumulated in stereocilia and vesicles that were pinched off from the plasma membrane. MIF detection in vesicles isolated from epididymal secretion together with the lack of a $\mathrm{N}$-terminal signal sequence for translocation in the endoplasmic reticulum strongly suggested a nonclassical secretion mode. Furthermore, MIF was identified as a new component of the outer dense fibers (ODF), a cytoskeletal element of the mid- and principal piece of the sperm tail.

Conclusion: The cytokine MIF was identified in substantial amounts in the epithelial cells of rat epididymis and in the outer dense fibers of rat epididymal spermatozoa. Our results indicate a nonclassical secretion mode for MIF and suggest a cell-to-cell transfer of MIF via vesicles to the sperm cells.

\section{Introduction}

Originally, the macrophage migration inhibitory factor (MIF) was described as a classical T cell cytokine $(1,2)$. In addition to its $\mathrm{T}$ cell-associated functions, MIF was identified as a pituitary hormone and macrophage-derived cytokine that counter-regulated the glucocorticoid-mediated suppression of the

Address correspondence and reprint requests to: Andreas Meinhardt, Ph.D., Department of Anatomy and Cell Biology, Philipps University of Marburg, Robert-Koch Str. 6, D-35037 Marburg, Germany. Phone: +49-6421-284021; Fax: +49-6421285783; E-mail: meinhar1@mailer.uni-marburg.de immune response $(3,4,5)$. Furthermore, MIF was detected as a key regulator in lipopolysaccaride (LPS)induced sepsis. Inhibition of MIF by neutralization using antibodies or by targeted gene disruption resulted in substantially increased survival rates of animals treated with otherwise lethal doses of lipopolysaccaride $(3,6)$. During the last few years, various additional properties of MIF have been discovered. MIF can function as an enzyme exhibiting tautomerase (7) and probably thiol-protein oxidoreductase activity (8). These data suggest that MIF has much broader roles beyond its function as a classical cytokine. This hypothesis is supported by the fact 
that MIF was found to be secreted by the Leydig cells of the testis, where it modulated inhibin production of Sertoli cells (9). Furthermore, MIF shows a unique compensatory production in rat testis. Depletion of the original MIF source, the Leydig cells, by the specific toxin, ethane dimethan sulfonate (EDS), caused a compensatory MIF expression by the Sertoli cells, which were negative in normal rat testis. Leydig cell repopulation of the interstitial tissue after EDS treatment by precursor cells resulted in a switch back to production by Leydig cells (10). The detection of MIF in the testis raised the possibility of the further existence of MIF in the other parts of the reproductive tract. In fact, Western blots analysis indicated the presence of a $12 \mathrm{kDa}$ protein in epididymal homogenates reacting with an antiMIF antibody. This prompted us to characterize the molecular nature of this immunoreactive $12 \mathrm{kDa}$ polypeptide in rat epididymis and in the epididymis duct fluid by using a combination of biochemical and molecular biological methods, as well as immunohistochemistry and in situ hybridization studies.

In mammals the composition of the epididymal fluid is largely maintained by a complex process of absorption and secretion of the epithelial cells lining the duct (11). The regulation of this process and the mechanisms of secretion are not well known, though a general agreement exists that the secreted compounds interact with the spermatozoa and are indispensable for the maturation of the latter (11).

\section{Materials and Methods}

\section{Reagents}

Reverse transcription-polymerase chain reaction (RTPCR) of peripheral blood monocytes was used to amplify the coding sequence of mouse MIF using primers 5'-CGCCATATGCCTATGTTCATCGTGAAC$3^{\prime}$ and 5'-CGGATCCGACTCAAGCGAAGGTGGAAC$3^{\prime}$, which incorporated the NdeI $\left(5^{\prime}\right)$ and BamHI (3') restriction sites. The cDNA originally was cloned into the pCRII vector (Invitrogen, Groningen, The Netherlands), subcloned into the NdeI and BamHI sites of the pET-17b vector (Novagen, Madison, WI), and then sequenced. Following the manufacturer's protocols, protein was expressed in pLysS cells and induced with $0.4 \mathrm{mM}$ isopropyl $\beta$-D-thiogalactoside for $3 \mathrm{hr}$ at $25^{\circ} \mathrm{C}$. Recombinant MIF (rec MIF) was then purified from the cell lysates. A monoclonal anti-mouse MIF antibody was raised in mouse using standard procedures.

\section{Tissue Preparation}

Male adult Wistar rats (ranging in body weight from 290-310 g; Charles River, Kisslegg, Germany) were anesthetized and killed by $\mathrm{CO}_{2}$ asphyxiation. Testes and epididymides were removed, and epididymal duct fluid was obtained separately from the caput, corpus, and cauda region by rinsing the respective epididymal segments in ice-cold buffer $(250 \mathrm{mM}$ sucrose, $10 \mathrm{mM}$ Tris-Cl, $1 \mathrm{mM}$ EDTA, and $1 \mathrm{mM}$ Pefabloc SC inhibitor; pH 7.4, Serva, Heidelberg, Germany). All successive steps were carried out at $4^{\circ} \mathrm{C}$. Epididymal sperm cells were separated at $600 \times$ $\mathrm{g}$ for $10 \mathrm{~min}$. The supernatant was centrifuged at $2,000 \times \mathrm{g}$ for $20 \mathrm{~min}$ to precipitate tissue fragments and cell debris. The resulting supernatant was used either for MIF isolation or was further ultracentrifuged at $100,000 \times \mathrm{g}$ for $30 \mathrm{~min}$ in order to isolate vesicles from epididymal duct fluid according to the method described by Fornes et al. (12). To obtain total tissue extracts decapsulated testes and the epididymides were processed carefully in Tris sucrose buffer using a Potter Elvehjem homogenizer (Braun Biotech, Melsungen, Germany). Tissue aggregates and cell debris were removed by centrifugation at $200 \times \mathrm{g}$ for $10 \mathrm{~min}$. The supernatants were collected and the cytosol separated from the cell organelles by ultracentrifugation at $100,000 \times \mathrm{g}$ for $30 \mathrm{~min}$. Protein content of the samples was analyzed according to the colorimetric method described by Bradford (13).

\section{SDS-PAGE}

Samples of testicular and epididymal cytosol, epididymal duct fluid, the vesicular fractions isolated from epididymal fluid, and isolated outer dense fiber (ODF) proteins from spermatozoa, respectively, were diluted 1:2 in reducing sample buffer containing $2 \%$ (weight per volume; w/v) SDS and $0.2 \%$ (w/v) DL-dithiothreitol (DTT). Samples were separated on a $10 \%$ Tricine-SDS polyacrylamide gel according to the method of Schagger and Jagow (14). Afterwards, the gels were fixed in $10 \%$ trichloroacetic acid (TCA) for $30 \mathrm{~min}$ and subsequently stained with silver (15) or Coomassie brillant blue.

\section{Western Blots Analysis}

After gel electrophoresis, proteins were transferred onto a $0.2 \mu \mathrm{m}$ nitrocellulose membrane (Schleicher \& Schuell, Dassel, Germany). The membrane then was incubated consecutively with a polyclonal rabbit antibody (1:800) raised against rec MIF overnight at RT and with peroxidase-conjugated goat anti-rabbit-immunoglobulin G (IgG) antibody (Cappel, Durham, NC; l:10,000) for $1 \mathrm{hr}$ at RT. Immunoreactive bands were visualized using the enhanced chemoluminescence (ECL) system (Amersham, Braunschweig, Germany) or, alternatively, with diaminobenzidine $(\mathrm{DAB}) / \mathrm{H}_{2} \mathrm{O}_{2}$.

\section{Purification of MIF From Rat Epididymis}

For purification of the $12 \mathrm{kDa}$ protein, epididymal fluid and cytosol fraction were fractionated by ammonium sulfate precipitation $(40-70 \%$ saturation). Precipitated proteins were redissolved in $50 \mathrm{mM}$ Tris-HCl buffer ( $\mathrm{pH}$ 7.5) containing 10 mM EDTA and further purified by anion exchange chromatography on a fast performance liquid chromatography 
(FPLC) system using a MonoQ-column (Pharmacia, Freiburg, Germany) equilibrated with $50 \mathrm{mM}$ Tris-HCl buffer (pH 7.5), 10 mM EDTA. Bound proteins were eluted using a gradient ranging from 0 to $1 \mathrm{M} \mathrm{NaCl}$ in $50 \mathrm{mM}$ Tris-HCl buffer (pH 7.5), $10 \mathrm{mM}$ EDTA. The $12 \mathrm{kDa}$ protein was detected in the first flow-through fractions by Western blots analysis. These fractions were pooled and applied to a Superdex TM 75 column (Pharmacia, Freiburg, Germany) equilibrated with $50 \mathrm{mM}$ Tris-HCl buffer ( $\mathrm{pH} \mathrm{7.8),}$ $1 \mathrm{mM}$ EDTA, and $100 \mathrm{mM} \mathrm{NaCl}$. Eluted fractions were tested for the $12 \mathrm{kDa}$ protein by Western blots analysis. Purity was verified by SDS-PAGE and silver staining.

\section{Amino Acid Sequence Analysis}

Fractions highly enriched with the $12 \mathrm{kDa}$ polypeptide were electrophoresed on a 10\% Tricine-SDS polyacrylamide gel as described above, then transferred onto polyvinylidene difluoride (PVDF) membrane (0.2 $\mu \mathrm{m}$; Bio-Rad, Munchen, Germany) according to the method of Kyhse-Andersen (16) using a semidry transfer unit (Serva, Heidelberg, Germany). After blotting, the membrane was stained with Coomassie brilliant blue for $1 \mathrm{~min}$, destained with $50 \%$ methanol, and then extensively washed with distilled water. The $12 \mathrm{kDa}$ band was excised and cut into small pieces. Amino terminal sequence analysis of the membrane bound polypeptide band was carried out by Edman degradation on an Applied Biosystems pulsed-liquid-phase sequencer, model $477 \mathrm{~A}$ (Foster City, CA). Phenylthiohydontoin (PTH) derivates of amino acids were identified by online detection on a model 120 A recorder (Applied Biosystems, Forster City, CA), with a repetitive yield of 92-95\%, according to the manufacturer's instructions.

\section{Reverse Transcription-Polymerase Chain Reaction (RT-PCR) and Cloning of cDNA Fragments}

Total RNA was extracted from rat testes and epididymides using the TrizolTM-reagent (Gibco BRL, Eggenstein, Germany) according to the manufacturer's instructions. $2 \mu \mathrm{l}$ aliquots of reverse transcribed cDNA were amplified by PCR using the following conditions: denaturation for $30 \mathrm{sec}$ at $94^{\circ} \mathrm{C}$, annealing for $30 \mathrm{sec}$ at $60^{\circ} \mathrm{C}$, and elongation for $1 \mathrm{~min}$ at $72^{\circ} \mathrm{C}$ using MIF-specific primers deduced from the published murine T-cell sequence (3) (25 cycles), or glyceraldehyde-3-phosphate dehydrogenase (GAPDH) primers (30 cycles) as a positive control. Murine MIF primers were 5'-CCATGCCTATGTTCATCGTG-3' and 5'GAACAGCGGTGCAGGTAAGTG-3' bordering a 381base pair (bp) segment. Primers for human and rat GAPDH were 5'-CGTCTTCACCACCATGGAGA-3' and 5'-CGGCCATCACGCCACAGTTT-3' bordering a 300-base pair segment. The eluted cDNA was then ligated into the pCR ${ }^{\mathrm{TM}}$ II cloning vector (Invitrogen, San Diego, CA) and sequenced by MWG Biotech (Ebersberg, Germany).

\section{Northern Blots Analysis}

$5 \mu \mathrm{g}$ of total RNA isolated from the rat testis and the separated caput, corpus, and cauda fractions of rat epididymis, respectively, were applied on a $1 \%$ agarose gel according to methods described by Sambrook et al. (17). Thereafter, transferred RNA was hybridized with digoxigenin (DIG)-labeled riboprobes (dilution of 1:5000) and detected using chemoluminescence reagents (Tropix, Heidelberg, Germany).

\section{In Situ Hybridization}

In situ hybridization was performed on $5 \mu \mathrm{m}$ paraffin sections of formalin-fixed tissue using a newly developed microwave-based protocol as described in detail by Lan et al. (18).

\section{Immunohistochemistry and Immunoelectron Microscopy}

Bouin's fixed tissue specimens were incubated overnight with the antibody raised against rec MIF. The bound antibody was visualized using the Universal LSAB 2 horseradish peroxidase kit according to the manufacturer's instructions (Dako, Hamburg, Germany). Sections were stained with diaminobenzidine (DAB) as the chromogenic substrate. For immunofluorescence analy-sis, isolated epididymal and testicular spermatozoa were treated with $1 \%$ Triton $\mathrm{X}-100$ in phosphate-buffered saline (PBS) for $15 \mathrm{~min}$. Primary anti-MIF antibody detection was performed using Cy3-fluorescence labeled secondary antibody (Dianova, Hamburg, Germany). Sperm cells were examined using a Zeiss LSM 410 invert laser scanning microscope.

For immunoelectron microscopy, small epididymal tissue fragments were fixed in a mixture of $0.5 \%$ glutaraldehyde, $1.25 \%$ paraformaldehyde, $0.025 \%$ picric acid, and $2.5 \%$ polyvinylpyrrolidine in $100 \mathrm{mM}$ cacodylate buffer ( $\mathrm{pH} 7.3$ ) overnight. Immu-noreaction on semithin sections and immunoelectron microscopy on ultrathin sections were performed as described previously by Steinhoff et al. (19). Antiserum pre-absorbed with rec MIF in excess and preimmune serum were used as negative controls. The vesicles isolated from epididymal fluid were fixed as above and processed for routine electron microscopical examination.

\section{Isolation of Outer Dense Fiber Proteins (ODF)}

The procedure followed the protocol developed by Oko (20), with modifications. Briefly, washed epididymal sperm from 20 rats were sonicated for 5 min in PBS (Labsonic U, B. Braun, Melsungen, Germany; 10W, repeating duty cycle: $0.5 \mathrm{sec}$, power level: maximal increased). Separated sperm tails and heads were pelleted and resuspended in $65 \%$ sucrose and layered over a sucrose step gradient composed of $8 \mathrm{ml}$ fractions of $65 \%$, $70 \%$, and $75 \%(\mathrm{w} / \mathrm{v})$ sucrose in water and centrifuged at $100,000 \times \mathrm{g}$ for $80 \mathrm{~min}$. The purified sperm tails 
were obtained from the interface between the supernatant and the $65 \%$ sucrose step. Isolated sperm tails were washed in PBS and then solubilized in $1 \%$ SDS, 2 mM DTT, 25 mM Tris- $\mathrm{HCl}$, pH 8.0 for $90 \mathrm{~min}$ at room temperature under constant shaking. The resulting solution $(12 \mathrm{ml})$ subsequently was layered over a second sucrose step gradient composed of $12 \mathrm{ml}$ fractions of $35 \%$ and $75 \%(\mathrm{w} / \mathrm{v}) \mathrm{su}-$ crose, and centrifuged at $100,000 \times \mathrm{g}$ for $60 \mathrm{~min}$. The ODF band obtained from the $35-75 \%$ interface was suspended in $25 \mathrm{mM}$ Tris- $\mathrm{HCl}, \mathrm{pH} \mathrm{8.0,} \mathrm{and} \mathrm{cen-}$ trifuged at $50,000 \times \mathrm{g}$ for $10 \mathrm{~min}$. The final pellet, as well as the supernatant obtained from the second gradient, was used for SDS-PAGE and Western blots analysis.

\section{Results}

\section{Detection of $12 \mathrm{kDa}$ Protein in the Epididymis}

The anti-MIF antiserum was raised to purified recombinant mouse MIF. Mouse MIF differs from rat MIF by only a single amino acid residue and antiMIF antiserum showed no cross-reactivity with other serum or tissue proteins. Western blots analysis of epididymal cytosol and duct fluid using the anti-MIF antibody revealed a single band at $12 \mathrm{kDa}$, which co-migrated with rec MIF (Fig. 1). MIF from testicular cytosol served as a further positive control $(9,10)$. The vesicular fraction isolated from epididymal secretion showed an identical band at $12 \mathrm{kDa}$ (Fig. 1). In general, the largest quantity of the $12 \mathrm{kDa}$ protein was detected in the cytosol and duct fluid from the caput region, respectively (Fig. 1). Substantially reduced immunoreaction was found in the cytosol and duct fluid from corpus and cauda region (Fig. 1) confirming the spatial distribution pattern also shown by immunohistochemistry (Fig. 2A-C).

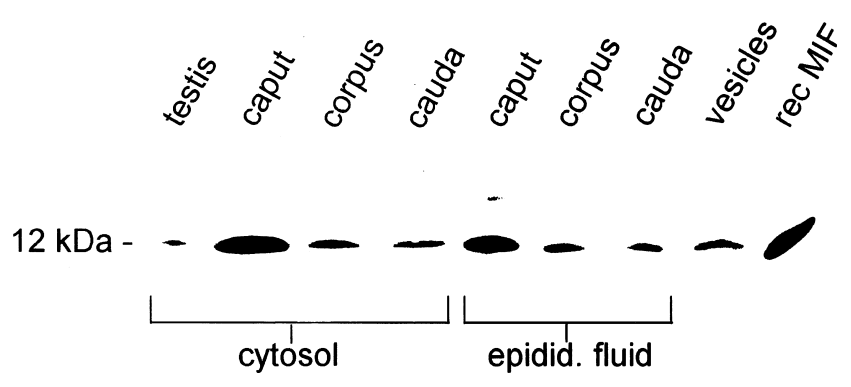

Fig. 1. Western blots analysis comparing rat testicular cytosol, epididymal cytosol (caput, corpus, cauda region), isolated epididymal fluid (caput, corpus, cauda region), and vesicles isolated from epididymal fluid ( $25 \mu \mathrm{g}$ per lane). Specimens were probed with the antibody directed against migration inhibitory factor (MIF). The immunoreactive band at $12 \mathrm{kDa}$ was comigrating with recombinant MIF (rec MIF, $2 \mu \mathrm{g}$ ). Highest amounts were detected in the epididymal cytosol and fluid fractions, with much lower quantities found in the corpus and cauda samples. The clear MIF band was also present in isolated vesicles from epididymal fluid.
Purification and Identification of the $12 \mathrm{kDa}$ Protein from Rat Epididymis

The $12 \mathrm{kDa}$ immunoreactive protein was purified from both rat epididymal cytosol and epididymal duct fluid in quantities sufficient for $\mathrm{N}$-terminal sequence analysis. Saturation of $40-70 \%$ ammonium sulfate resulted in the precipitation of the 12 kDa-immunoreactive protein and in about 5 -fold enrichment. Anion exchange chromatography on a MonoQ-column showed a further accumulation of the $12 \mathrm{kDa}$ protein in the unbound fraction (data not shown). Subsequent gel exclusion chromatography on a Superdex 75 column yielded highly enriched preparations of the epididymal protein, as shown by SDS-PAGE and subsequent silver staining (Fig. 3A), and by Western blots analysis (Fig. 3B). Compared with the elution volumes of the reference proteins albumin $(66.0 \mathrm{kDa})$, trypsinogen $(24.0 \mathrm{kDa})$, and $\alpha$-lactalbumin (14.2 kDa), the elution volume of MIF confirmed the relative molecular mass $\left(\mathrm{M}_{\mathrm{r}}\right)$ of $12 \mathrm{kDa}$ for monomeric epididymal MIF. N-terminal analysis of 10 amino acids of the purified $12 \mathrm{kDa}$ polypeptide revealed complete homology to the $\mathrm{N}$ terminal sequence of the cytokine MIF (21) as shown in Fig. 3C.

\section{Immunohistochemical Localization of MIF}

MIF labeling was clearly visible in the epithelium of rat epididymis (Fig. 2A-B). Principle cells were the predominant positively staining cell type in the epithelium; whereas, a certain proportion of the basal cells were negative (Fig. 2A-B). A spatial distribution pattern of MIF was visible with the strongest immunoreaction found in the epithelium of the caput region. Substantially lower levels were seen in the corpus and cauda region. No staining was evident in the stromal part of the epididymis, including all macrophages (Fig. 2A-B). Sections showed no staining after pre-incubation of the antiserum with excess rec MIF or after using preimmune serum instead of the MIF antibody (Fig. 2C). In isolated epididymal spermatozoa, MIF-immunofluorescence labeling was seen with high intensity in the midpiece of sperm tails; whereas, sperm heads were clearly negative (Fig. 4). It is of note, that pre-treatment of sperm with Triton X-100 was required to reveal MIF immunoreactivity in the spermatozoa.

\section{Expression of MIF mRNA in the Rat Epididymis}

RT-PCR of RNA from both rat testes and epididymis resulted in the amplification of a single product that was consistent with the expected length $(381 \mathrm{bp})$ of rat MIF (data not shown). Subsequent sequencing of the entire cloned fragment (381 bp out of $519 \mathrm{bp}$ of the full length MIF cDNA) resulted in a $100 \%$ homology between the RT-PCR amplified fragment from rat epididymis and the previously published rat MIF cDNA from rat liver (19) (data not shown). 

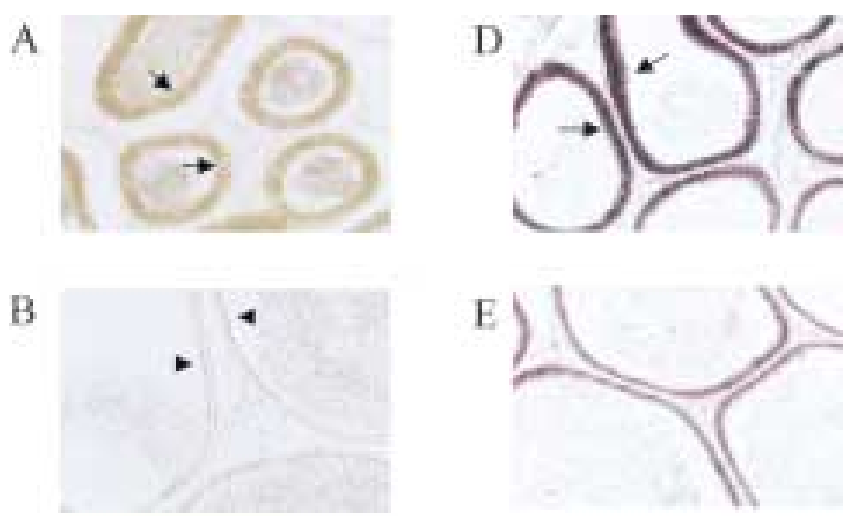

C

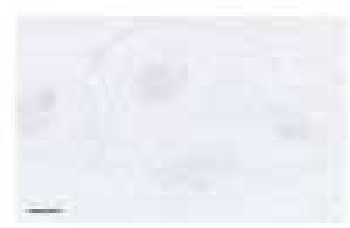

F

complex were negative for MIF (Fig. 6B,C). MIF accumulation was evident in the stereocilia and vesicles pinched off from the apical cell surface of the epithelium (Fig. 6B).

Strikingly, a dense colloidal gold labeling was apparent in the ODF of luminal spermatozoa (Fig. 6A). The fibrous sheath in the principal piece, a further structural component of the sperm tail, and the mitochondria wrapped around the axoneme showed only faint background staining. Vesicles seen in close association with the sperm tail also were positive for MIF (Fig. 6A). The vesicles seen in the intact epididymis resembled structurally those isolated from the duct fluid (Fig. $6 \mathrm{E}$ ) and clearly did not comprise cross-sectioned stereocilia.

Vesicles separated from rat epididymal fluid, which were shown to contain substantial amounts of MIF using Western blots (Fig. 1) and immunoelectron microscopy (Fig. 6D), consisted mainly of a heterogeneous population of membrane-bound structures. The vesicles were roughly rounded in

Fig. 2. Immunohistochemistry (A-C) and in situ hybridization (D-F) of MIF in rat epididymis. (A) Caput epididymis. Strong migration inhibitory factor (MIF) immunoreaction is visible in the epithelial cells (arrows); whereas, the stroma is negative. (B) In the cauda region, MIF labeling in epithelial cells is clearly less intense. Note the predominant apical staining of the epithelium (arrowheads). (C) Negative control using anti-MIF antibody preabsorbed with recombinant MIF (rec MIF). (D) Caput region. In situ hybridization shows strong MIF mRNA expression in the epithelial cells (arrows). Again, the stroma is negative. (E) Corresponding to the immunohistochemical results, the cauda region shows substantially weaker MIF expression. (F) Sense negative control, caput region. Bar $=40 \mu \mathrm{m}$.

Northern blots analysis of rat testis (control), caput, corpus, and cauda epididymis showed a differential MIF expression (Fig. 5A). The strongest MIF mRNA level was found in the caput region; whereas, the more distal parts of the epididymis were less pro- nounced (Fig. 5A), confirming the previously mentioned regional distribution pattern. RNA amounts were assessed by both spectrophotometry and ethidium bromide staining of the respective gel (Fig. 5B).

In situ hybridization clearly localized MIF mRNA to the epithelium of rat epididymis; whereas, the stromal part was not labeled (Fig. 2D-F). MIF mRNA was expressed in a regionally distributed manner, with the highest intensity seen in the caput region. The labeling of the epithelial cells of the corpus and cauda part was substantially weaker. In contrast to the principal cells, basal cells of the epithelium revealed no MIF expression (Fig. 2D-F).

\section{Immunoelectron Microscopical Localization of MIF}

For ultrastructural localization of MIF, ultrathin sections of rat epididymis were incubated with antiMIF antibody. Specific labeling with gold particles was observed in the cytoplasmic compartment; whereas, the endoplasmic reticulum and the Golgi

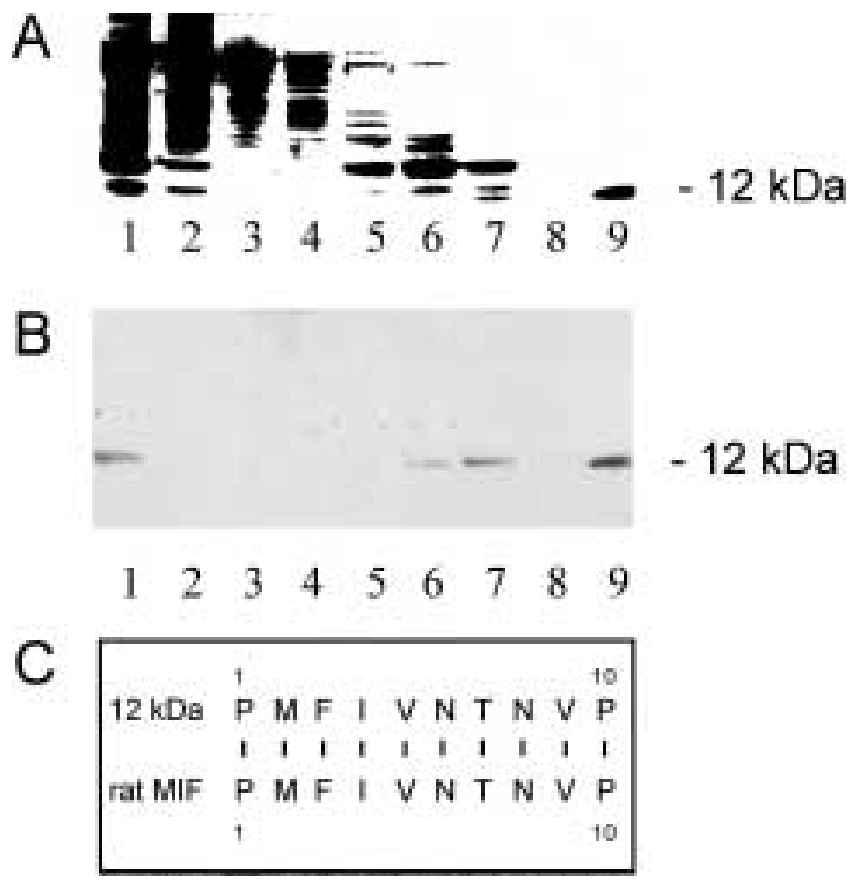

Fig. 3. Purification of MIF from rat epididymis. Fractions eluted from the Superdex 75 column were separated using (A) $10 \%$ Tricine SDS-PAGE with subsequent silver nitrate staining and (B) parallel Western blots analysis using the antimigration inhibitory factor (MIF) antibody illustrating the MIF purification steps. Lane 1: MIF containing material applied to the Superdex 75 column $(25 \mu \mathrm{g})$; lane 2: fraction $10(25 \mu \mathrm{g})$; lane 3: fraction $11(25 \mu \mathrm{g})$; lane 4: fraction $12(25 \mu \mathrm{g})$; lane 5: fraction $13(21 \mu \mathrm{g})$; lane 6: fraction $14(18 \mu \mathrm{g})$; lane 7 : fraction $15(5 \mu \mathrm{g})$; lane 8: fraction $19(2 \mu \mathrm{g}) ;(2-8$ fractions eluted from Superdex 75); lane 9: rec MIF $(0.5 \mu \mathrm{g})$. (C) Alignment of the $\mathrm{N}$-terminal amino acid sequence of the purified $12 \mathrm{kDa}$ protein with the N-terminal sequence of rat MIF (Sakai et al., 1994) revealed a $100 \%$ sequence identity. 


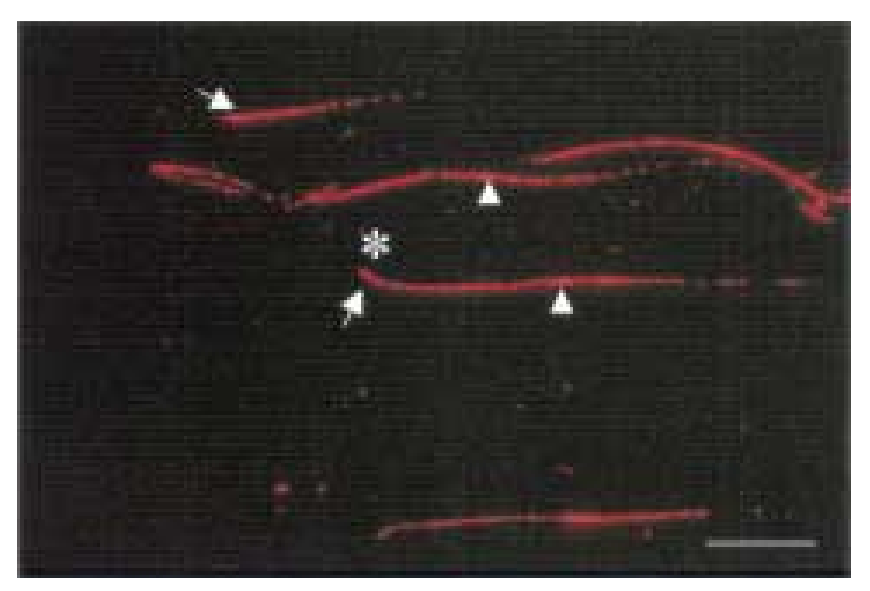

Fig. 4. Laser scanning micrograph of MIF-immunofluorescence labeling in isolated epididymal spermatozoa. Several spermatozoa are visible. Triton X-100 pretreatment was required to obtain a signal. Migration inhibitory factor (MIF) is localized in the sperm midpiece (arrows) and principal piece (arrowheads); whereas, the sperm heads (asterix) and the distal end of the sperm tail are clearly negative. The MIF distribution correlates with the localization of the outer dense fibers (ODF) in spermatozoa. Bar $=10 \mu \mathrm{m}$.

shape and varying in diameter. Their content was electron-lucent, however, and the smaller vesicles were seen to contain a more opaque granular material (Fig. 6E). Only a few contaminating mitochondria were observed in this fraction.

\section{MIF as a Component of the ODF of Rat Spermatozoa}

SDS-PAGE of the isolated ODF fraction revealed a series of protein bands. Those around $80-85 \mathrm{kDa}$, a complex around $60-70 \mathrm{kDa}, 47 \mathrm{kDa}$, and 23-32 $\mathrm{kDa}$ were the most prominent bands (Fig. 7A). A minor band was seen at around $12 \mathrm{kDa}$. Using Western blots analysis, the anti-MIF antiserum reacted strongly with a band at $12 \mathrm{kDa}$ in the ODF fraction, as well as with total epididymal sperm from the caput and cauda region (Fig. 7B). A much weaker reaction was seen in the supernatant collected after the second gradient purification step, which contained other proteins solubilized from the sperm tails (Fig. 7B). This clearly showed that MIF protein was a component of the ODF of rat epididymal spermatozoa.

\section{Discussion}

Our Western blots analysis of rat epididymal homogenates reveals an immunoreactive band at $12 \mathrm{kDa}$ comigrating with recombinant MIF, which is much more intense, compared with that obtained from testicular homogenates. As cytokine expression occurs, usually at much lower levels, and epididymal proteins often display, organ-specific modifications, we characterize this immunoreactive $12 \mathrm{kDa}$ band of the epididymis at the molecular level.
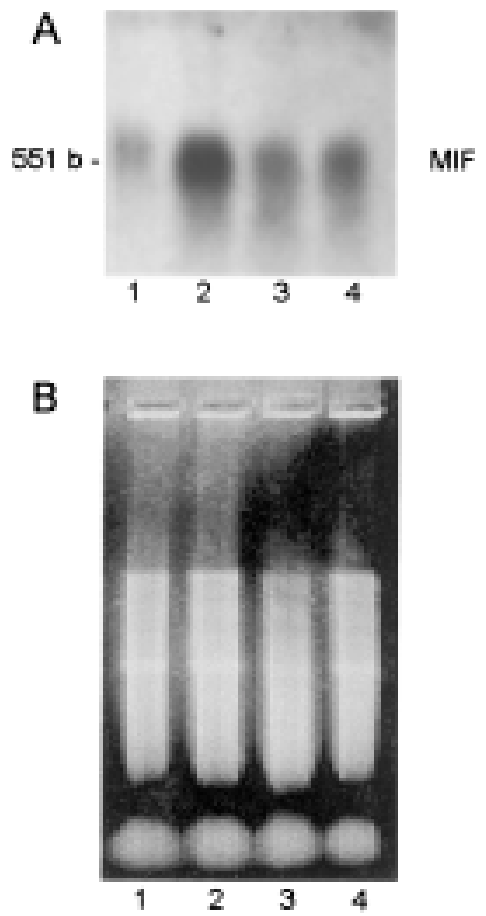

Fig. 5. Expression of MIF mRNA in the rat epididymis. (A) Northern blots analysis using a digoxigenin-labeled cRNA probe derived from the reverse transcription-polymerase chain reaction (RT-PCR) fragment. Migration inhibitory factor (MIF) mRNA shows a spatial expression pattern, with the highest amounts in the caput region. (B): The respective RNA gel stained with ethidium bromide is serving as a loading control. Lane 1: rat testis; lane 2: rat epididymis caput region; lane 3: epididymis corpus region; lane 4: epididymis cauda region.

Consecutive ammonium sulfate precipitation, anion exchange chromatography on MonoQ, and gel filtration chromatography on Superdex 75 yields a highly enriched preparation of the $12 \mathrm{kDa}$ polypeptide, which is eluted as a monomer. The subsequent $\mathrm{N}$-terminal amino acid sequence analysis reveals a $100 \%$ homology over 10 amino acids to the published N-terminus of the cytokine MIF from rat (21). This data was confirmed at the RNA level by sequence analysis of a RT-PCR amplified cDNA fragment from rat epididymis, which resulted in $100 \%$ identity with the published rat MIF cDNA sequence (21). These results clearly demonstrate that MIF mRNA and protein are synthesized by the rat epididymis.

We hypothesized that the substantial amounts of MIF mRNA and protein detected by Northern and Western blots analysis, respectively, could not be explained solely by immune cell production. Therefore, we performed both immunohistochemistry and in situ hybridization to investigate the source of MIF production in the epididymis. The combination of both methods is particularly important as immunohistochemistry alone shows only the presence of an antigen, which in case of the cytokine MIF also can be the site of binding or uptake. Both approaches 

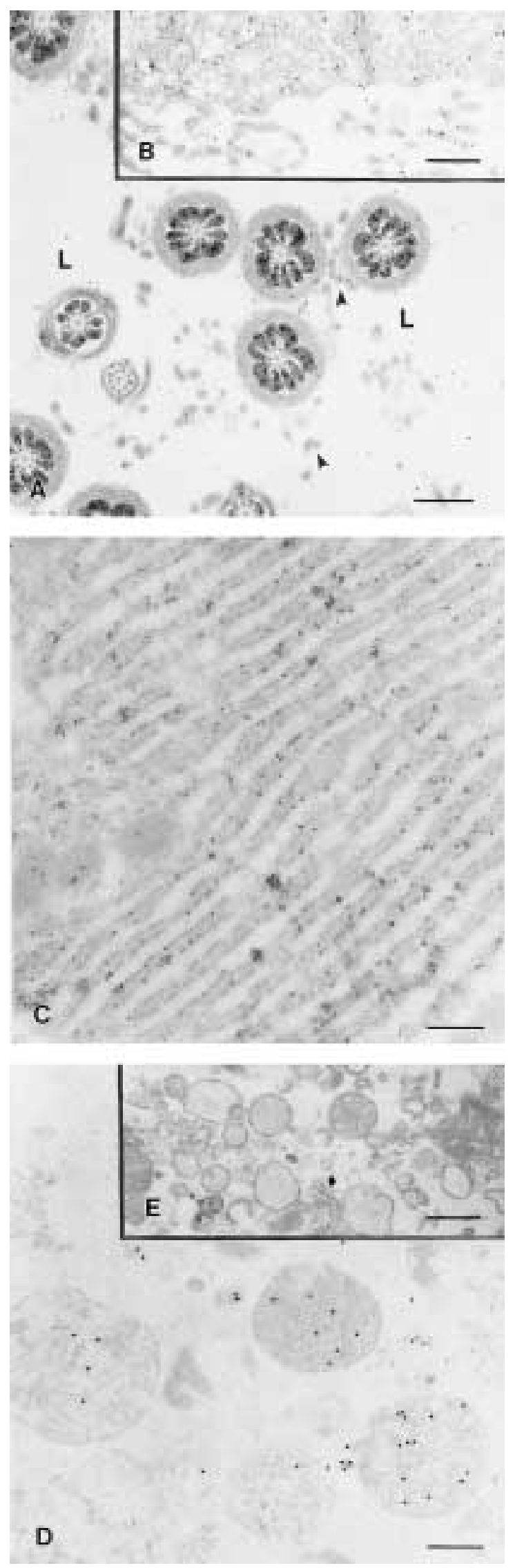

demonstrate that MIF is synthesized by the epithelial cells of the rat epididymis with the principal cells as the main source. In contrast, most of the epithelial basal cells show no evidence of MIF expression. The stromal compartment, including all macro-phages, is completely devoid of any MIF labeling. Therefore, MIF is the first classical cytokine identified to be synthesized by the epithelial cells of the epididymis. Its detection in vesicles isolated from epididymal fluid and the lack of a $\mathrm{N}$-terminal signal sequence for translocation into the endoplasmic reticulum (ER) is strongly suggestive of a nonclassical mode of secretion. To further investigate this possibility, we performed immunoelectron microscopy, which found MIF localizes in the cytoplasm of epididymal epithelial cells with no gold-labeling visible in the ER cisternae and the Golgi complex. At the luminal cell surface MIF, is found in stereocilia and in vesicles released from the apical plasma membrane. A similar secretion mode, the apocrine secretion, recently was described in the rat epididymis for carbonic anhydrase II (CAH II) (22) and for the vas deferens (23). Furthermore, apocrine secretion for CAH II (24) and for secretory transglutaminase (TGase) $(25,19)$ was reported in the coagulating gland, an accessory sex gland of male rats. CAH II and TGase, like MIF, do not contain an ER signal sequence, are located exclusively in the cytoplasm, and bind to albumin (26), which was identified as a likely carrier of proteins destined for apocrine secretion (27). The MIFcontaining vesicles in epididymal fluid suggest a possible cell-to-cell transfer of MIF to the spermatozoa. A similar type of transfer from the epididymal epithelium to sperm cells was shown for the human epididymal (HE) product, HE5, a small glycosylphosphatidylinositol-anchored glycopeptide secreted by the epididymal principal cells $(28,29)$. This mechanism could be mediated by the intimate contact between vesicles in epididymal duct fluid and spermatozoa, demonstrated at the ultrastructural level by the findings of Fornes and de Rosas (30). Indeed, in our study, MIF-positive vesicles also are seen in close association with the sperm tail. The hy-

Fig. 6. Immunoelectron microscopy of MIF in the caput region of the rat epididymis. (A) High power magnification shows the specific migration inhibitory factor (MIF) staining of the outer dense fibers (ODF). Note MIF-positive vesicles (arrowhead) around the spermatozoa. (Bar $=60 \mathrm{~nm}$ ). $\mathrm{L}=$ epididymal lumen. (B) The apical cell pole of an epithelial cell in rat caput epididymis. Stereocilia and vesicles pinched off from the apical plasma membrane show accumulation of MIF. (Bar $=50$ $\mathrm{nm}$ ). (C) Gold-labeling is restricted to the cytoplasm of the epididymal epithelium (dark regions). The endoplasmic reticulum is clearly negative for MIF (bright regions). (Bar $=50 \mathrm{~nm}$ ). (D) Immunoelectron microscopy using the anti-MIF antibody shows the presence of this peptide in vesicles isolated from epididymal fluid. (Bar $=7 \mathrm{~nm}$ ). (E) Isolated vesicles examined using conventional transmission electron microscopy demonstrate a heterogeneous membrane-bound population similar to vesicles seen in the intact organ. $(\mathrm{Bar}=20 \mathrm{~nm})$. 


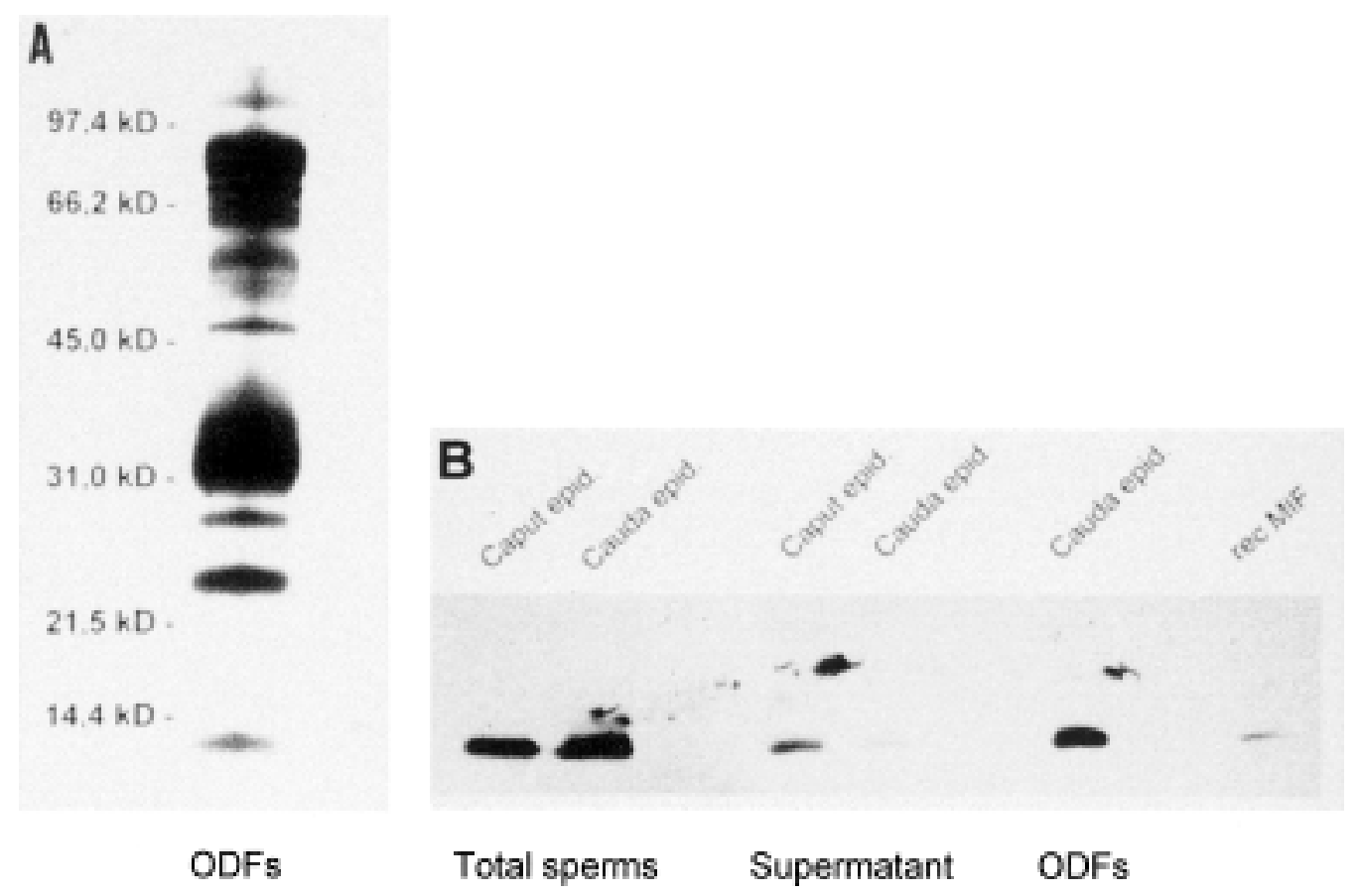

Fig. 7. Detection of MIF in the ODF of spermatozoa. (A) SDS-PAGE with subsequent Coomassie brillant blue staining of the purified outer dense fiber (ODF) proteins from epididymal spermatozoa. Characteristically are the prominent bands at around 80-85 kDa, a complex around 60-70 kDa, $47 \mathrm{kDa}$, and 23-32 kDa. A minor band was seen at around $12 \mathrm{kDa}$. (B) Western blots analysis of total sperm from caput and cauda epididymis, and isolated outer dense fiber (ODF) proteins obtained from epididymal spermatozoa show a strong immunoreactive band at $12 \mathrm{kDa}$ when probed with the anti-MIF antibody. The supernatant yield from the second gradient centrifugation step, which is used to separate the ODF from other tail proteins, contains markedly less migration inhibitory factor (MIF). Recombinant MIF (rec MIF; $0.5 \mu \mathrm{g}$ ) was used as a positive control.

pothesis of a cell-to-cell transfer of MIF is further supported by the fact that MIF, clearly visible in the midpiece and principal piece of epididymal spermatozoa pretreated with Triton $\mathrm{X}-100$, is not seen in spermatozoa in the testis $(9,10)$. More importantly, using immunoelectron microscopy, a strong MIFlabeling is evident in the outer dense fibers (ODF) of the sperm tail. High power micrographs reveal that most of the MIF protein is confined to the cortex of the ODF. The ODF are a cytoskeletal element that surround the axoneme in the middle piece of the flagellum, which is characterized by the presence of the mitochondrial sheath, and in the more distal principal piece. In the middle piece there are nine ODF, each one connects with one microtubular doublet of the axoneme. In the principal piece, the ODF are reduced to seven and progressively decrease in size distally (31). Biochemical analysis show that the ODF proteins identified so far are cystein-rich proteins. These proteins successively form disulfide bonds during epididymal passage, a step crucial for providing the sperm tail with the indispensable stiffness for the generation of sperm motility, especially progressive motility $(32,33)$. Despite the importance of the timely formation of the disulfide bonds in the ODF for male fertility, the molecular basis of this alteration is poorly understood.
As a conventional membrane receptor for MIF has not been identified, other nonreceptor-mediated effector mechanisms must be considered. One such mechanism recently was described by Kleemann et al. (8), who found that MIF can function in vitro as an enzyme exhibiting thiol-protein oxidoreductase activity. This is particularly interesting in light of the striking presence of MIF in the ODF of the sperm tail. This colocalization could provide the molecular basis for an enzymatic role of MIF in this process. Recent findings of Ursini et al. (34) raise an alternative structural function for MIF. Their study showed that the selenoprotein phospholipid hydroperoxide glutathione peroxidase (PHGPx) could change function and physicochemical characteristics. During sperm maturation, PHGPX switched from a soluble active enzyme to an enzymatically inactive structural protein of the sperm tail (34).

Taken together, we identified MIF on the molecular and cellular level as the first classical cytokine to be expressed and secreted by the rat epididymal epithelium. Our data support the view that MIF is secreted in a nonclassical secretion mode via apocrine-released vesicles into the epididymal fluid where MIF associates with the ODF of the sperm midpiece. 


\section{Acknowledgements}

The authors wish to thank Mrs. Claudia Keppler, Mrs. Gudrun Hoffbauer, and Mr. Gerhard Jennemann for expert technical support. We are grateful to Dr. Con Mallidis (UCLA) for critically reading the manuscript and his valuable comments. This work was supported by a grant from the Deutsche Forschungsgemeinschaft (Me 1323/2-1) and the Fonds der Chemischen Industrie to A.M.

\section{References}

1. David JR. (1966) Delayed hypersensitivity in vitro. Its mediation by cell-free substances formed by lymphoid cell-antigen interaction. Proc. Natl. Acad. Sci. USA 56: 72-77.

2. Bloom BR, Bennett B. (1966) Mechanism of a reaction in vitro associated with delayed-type hypersensitivity. Science 153: 80-82.

3. Bernhagen J, Calandra T, Mitchell RA, et al. (1993) MIF is a pituitary derived cytokine that potentiates lethal endotoxaemia. Nature 365: 756-759.

4. Calandra T, Bernhagen J, Mitchell RA, Bucala R. (1994) The macrophage is an important and previously unrecognized source of macrophage migration inhibitory factor. J. Exp. Med. 179: $1895-1902$.

5. Calandra T, Bernhagen J, Metz CN, et al. (1995) MIF as a glucocorticoid-induced modulator of cytokine production. Nature 377: 68-71.

6. Bozza M, Satoskar AR, Lin G, et al. (1999) Targeted disruption of migration inhibitory factor gene reveals its critical role in sepsis. J. Exp. Med. 189: 341-346.

7. Rosengren E, Aman P, Thelin S, et al. (1997) The macrophage migration inhibitory factor MIF is a phenylpyruvate tautomerase. FEBS Lett. 417: 85-88.

8. Kleemann R, Kapurniotu A, Frank RW, et al. (1998) Disulfide analysis reveals a role of macrophage migration inhibitory factor (MIF) as thiol-protein oxidoreductase. J. Mol. Biol. 280: 85-102.

9. Meinhardt A, Bacher M, R. McFarlane, JR., et al. (1996) Macrophage migration inhibitory factor production by Leydig cells: evidence for a role in the regulation of testicular function. Endocrinology 137: 5090-5095.

10. Meinhardt A, Bacher M, R. McFarlane, JR., et al. (1999) A switch in the cellular localization of macrophage migration inhibitory factor in the rat testis after ethane dimethane sulfonate treatment. J. Cell Sci. 112: 1337-1344.

11. Kirchhoff C. (1999) Gene expression in the epidiymis. Int. Rev. Cytol. 188: 133-202.

12. Fornes MW, Barbieri A, Sosa A, Bertini F. (1991) First observations on enzymatic activity and protein content of vesicles separated from rat epididymal fluid. Andrologia 23: 347-351.

13. Bradford M. (1976) A rapid and sensitive method for the quantification of microgram quantities of protein utilizing the principle of protein-dye binding. Anal. Biochem. 72: 248-254.

14. Schagger H, von Jagow G. (1987) Tricine-sodium dodecyl sulfate-polyacrylamid gel electrophoresis for the separation of proteins in the range from 1 to $100 \mathrm{kDa}$. Anal. Biochem. 166: 368-379.

15. Blum H, Beier H, Groos, HJ. (1987) Improved silver staining of plant proteins, RNA and DNA in polyacrylamide gels. Electrophoresis 8: 93-99.
16. Kyhse-Andersen J. (1984) Electroblotting of multiple gels: a simple apparatus without buffer tank for rapid transfer of proteins from polyacrylamide to nitrocellulose. J. Biochem. Biophys. Methods 10: 203-209.

17. Sambrook J, Fritsch EF, Maniatis T. (1989) Molecular Cloning: A Laboratory Manual, 2nd Ed. Cold Spring Harbor Laboratory, Cold Spring Harbor, NY, pp 199

18. Lan HY, Mu W, Ng YY, Nikolic-Paterson DJ, Atkins RC. (1996) A simple, reliable, and sensitive method of nonradioactive in situ hybridization: use of microwave heating to improve hybridization efficiency and preserve tissue morphology. J. Histochem. Cytochem. 44: 281-287.

19. Steinhoff M, Eicheler W, Holterhus PM, Rausch U, Seitz J, Aumüller G. (1994) Hormonally induced changes in apocrine secretion of transglutaminase in the rat dorsal prostate and coagulating gland. Eur. J. Cell Biol. 65: 49-59.

20. Oko R. (1988) Comparative analysis of proteins from the fibrous sheath and outer dense fibers of rat spermatozoa. Biol. Reprod. 39: 169-182.

21. Sakai M, Nishihira J, Hibiya Y, Koyama Y, Nishi S. (1994) Glutathione binding rat liver 13k protein is the homologue of the macrophage migration inhibitory factor. Biochem. Mol. Biol. Int. 33: 439-446.

22. Kaunisto K, Parkkila S, Tammela T, Rönnberg L, Rajaniemi H. (1990) Immunohistochemical localization of carbonic anhydrase isoenzymes in the human male reproductive tract. Histochemistry 94: 381-386.

23. Manin M, Lecher P, Martinez A, Tournadre S, Jean C. (1995) Exportation of mouse vas deferens protein, a protein without a signal peptide, from mouse vas deferens epithelium: a model of apocrine secretion. Biol. Reprod. 52: 50-62.

24. Wilhelm B, Keppler C, Hoffbauer G, et al. (1998) Cytoplasmic carbonic anhydrase II of rat coagulating gland is secreted via the apocrine export mode. J. Histochem. Cytochem. 46: 505-511.

25. Seitz J, Keppler C, Rausch U, Aumüller G. (1990) Immunohistochemistry of rat secretory transglutaminase from rodent prostate. Histochemistry 93: 525-530.

26. Zeng FY, Kratzin H, Gabius HJ. (1994) Migration inhibitory factor-binding sarcolectin from human placenta is indistinguishable from a subfraction of human serum albumin. Biol. Chem. Hoppe Seyler 375: 393-399.

27. Wilhelm B, Meinhardt A, Renneberg H, et al. (1999) Serum albumin as a potential carrier for apocrine secreted proteins in the rat coagulating gland. Eur. J. Cell Biol. 78: 256-264.

28. Kirchhoff C. (1994) A major messenger ribonucleic acid of the rodent epididymis encodes a small glycosylphosphatidylinositol-anchored lymphocyte surface antigen. Biol. Reprod. 50: 896-902.

29. Kirchhoff C, Hale G. (1996) Cell-to-cell transfer of glycosylphosphatidylinositol-anchored membrane proteins during sperm maturation. Mol. Hum. Reprod. 2: 177-184.

30. Fornes MW, de Rosas JC. (1991) Interaction between rat epididymal epithelium and spermatozoa. Anat. Rec. 231: 193- 200.

31. Fawcett DW. (1975) The mammalian spermatozoon. Dev. Biol. 44: 394-436.

32. Calvin HI, Bedford JM. (1971) Formation of disulphide bonds in the nucleus and accessory structures of mammalian spermatozoa during maturation in the epididymis. J. Reprod. Fertil. Suppl. 13: 65-75.

33. Seligman J, Shalgi R. (1991) Protein thiols in spermatozoa and epididymal fluid of rats. J. Reprod. Fertil. 93: 399-408.

34. Ursini F, Heim S, Kiess M, et al. (1999) Dual function of the selenoprotein PHGPX during sperm maturation. Science 285: 1393-1396. 